\title{
Artikel
}

Simon Meier-Vieracker*

\section{Wutreden und andere invektive Gattungen zwischen Rekonstruktion und Aneignung}


Abstract: This paper deals with 'Wutreden' (rants) as an invective genre in digital media. It is argued that the generic aspects of rants are not due to the formal and functional features of the speech events alone, but should be described as the result of the practices of doing genre. Digital media with its affordances to recontextualization and serialization allow to reframe disparate speech events as instances of one generic scheme. As a result, the emerging concept of rants as a genre enables the production of new instances. In order to grasp this genre in the making, a discursive concept of genre is needed, which then can also be applied to other invective genres such as shitstorms and hate facts.

Keywords: Wutreden, Shitstorms, Hate Facts, Doing Genre, Digitale Medien, Diskurs, Rekontextualisierung - rants, shitstorms, hate facts, doing gender, digital media, discourse, recontextualization

*Prof. Dr. Simon Meier-Vieracker, TU Dresden, Institut für Germanistik, Professur für Angewandte Linguistik, simon.meier-vieracker@tu-dresden.de

\section{Einleitung}

Thema des vorliegenden Aufsatzes ist die Frage nach der Ausbildung und Transformation invektiver Gattungen oder, etwas präziser formuliert, die Frage danach, wie insbesondere im Kontext digitaler Medien invektive Dynamiken durch sich herausbildende Gattungen geordnet und umstrukturiert werden. Empirischer Ausgangspunkt ist dabei die Beobachtung, dass in den letzten Jahren immer häufiger Redeereignisse eines bestimmten Typs mediale Aufmerksamkeit erlangen und dabei als "Wutreden" bezeichnet werden. Damit einher geht aber auch eine zunehmende Subsumierung verschiedenster Redeereignisse unter diesen Terminus. Noch vor zwanzig Jahren haben nur Fußballtrainer Reden gehalten, die als "Wutreden" bezeichnet worden sind; besonders prominent natürlich Giovanni Trapattoni in seiner berühmten Pressekonferenz im Jahr 1998, die mit ihren Formulierungen wie "Flasche leer" oder "Was erlaube Strunz" längst in das allgemein verfügbare Schimpfinventar übergegangen ist. Inzwischen halten aber auch Politiker wie Christian Lindner oder Cem Özdemir
,Wutreden', und auch der Literaturkritiker Marcel Reich-Ranicki und - wenn man den Schlagzeilen Glauben schenken mag - sogar Papst Franziskus haben sich als Wutredner hervorgetan. ${ }^{1}$ Bei Greta Thunbergs Ansprache vor den Vereinten Nationen im September 2019 war man sich zumindest in der deutschsprachigen Presse ebenfalls schnell einig, dass es sich um eine Wutrede gehandelt habe. $^{2}$ Pressekonferenzen, Predigten, Telefoninterviews, Dankesreden, aber auch InstagramStories, Facebook-Posts, Zeitungskolumnen und sogar ganze Romane können inzwischen als Wutreden bezeichnet werden.

Mit dem Ausdruck ,Wutrede' liegt also eine Ethnokategorie zur benennenden Klassifizierung verschiedenartiger, invektiv konturierter Redeereignisse vor. Diese Kategorie ist Produkt und Vollzugsform einer Typisierungspraxis, wie sie in neueren linguistischen Forschungen typischerweise auch für wissenschaftliche Textsorten- und Gattungstypologien den Ausgangspunkt bildet. $^{3}$

1 Tsp/rtr/epd/KNA (2014) Wutrede von Papst Franziskus. 2 o.V. (2019) Thunberg hält Wutrede bei UN.

3 Habscheid (2011) Das halbe Leben, S. 14-17. 
Trotzdem versperrt sich die Wutrede den üblichen Zugriffen auf Gattungen in der Linguistik wie auch in der Soziologie, wo diese typischerweise als Bündel formal-funktionaler Elemente aufgefasst werden, die sich als Reflexe wiederkehrender kommunikativer Aufgaben verfestigt haben. ${ }^{4}$ Das Beispiel der Wutreden zeigt vielmehr, dass auch jenseits eines solchen formalfunktional bestimmbaren Substrats Gattungszusammenhänge konstruiert werden können. Um das Phänomen der Wutreden in gattungstheoretischer Perspektive angemessen beschreiben zu können, bedarf es deshalb eines rezeptions- und diskursorientierten Gattungsbegriffs, der stärker auf die metapragmatischen Rahmungen abzielt, durch die disparate Diskursereignisse als zusammengehörig gerahmt und dadurch interpretativ vorstrukturiert werden.

Ein solcher rezeptionsorientierter Gattungsbegriff hat sich besonders in neueren gattungstheoretischen Forschungen zu digitalen und sozialen Medien als hilfreich erwiesen. ${ }^{5}$ Denn hier lassen sich die metapragmatischen Rahmungen, die typischerweise mit intermedialen Rekontextualisierungen einhergehen, besonders detailliert und in ihrem Vollzugscharakter empirisch beobachten. Sie weisen aber auch über die digitalen Medien hinaus, indem sie das einschlägige Gattungswissen für den kommunikativen Haushalt insgesamt verfügbar machen und schließlich die Produktion neuer Gattungsexemplare anleiten können. Das Beispiel der Wutreden zeigt somit, wie vielfältig die Deutungsmöglichkeiten und Deutungsmodi von Invektiv-Geschehen ${ }^{6}$ gerade im Bereich der digitalen Medien sein können und welche Effekte der diskursiven Ordnung sich hieraus ergeben. Dabei handelt es sich bei den Wutreden zwar um einen besonders deutlichen, aber nicht singulären Fall von Gattungskonstruktion im Bereich des Invektiven. An verwandten internettypischen Phänomenen wie ,Shitstorms' und ,Hate Speech' kann gezeigt werden, wie sich ursprünglich rekonstruktive Kategorien herabsetzender Rede zusehends in produktive Schablonen zu wandeln scheinen, die sich Akteure gezielt aneignen.

4 Reisigl (2014) Gattung; Knoblauch/Schnettler (2010) Sozialwissenschaftliche Gattungsforschung.

5 Lomborg (2011) Social Media.

6 Ellerbrock/Koch/Müller-Mall et al. (2017) Invektivität, S. 6.
Im Folgenden werde ich zunächst darstellen, was man unter Wutreden versteht (2) und inwiefern man Wutreden als invektive Gattung beschreiben kann (3). Die sich hier auftuenden Probleme werde ich dann zum Anlass nehmen, einen rezeptions- und diskursorientierten Gattungsbegriff zu entwickeln, der über die Gattungsexemplare hinaus vor allem Praktiken des Doing Genre fokussiert, und werde zeigen, welche analytischen Perspektiven ein solcher Gattungsbegriff gerade angesichts der medialen Bedingungen des Internets eröffnet (4). Danach werde ich neuere Entwicklungen diskutieren, die zeigen, wie sich Akteure die Kategorie der Wutrede produktiv aneignen, so dass sie als verfügbare Schablone im Haushalt des Invektiven zusehends verankert wird (5). Anschließend werde ich mit Shitstorms und Hate Facts verwandte Fälle diskutieren, die sich vor allem in politischen Kontexten in ähnlicher Weise zwischen Rekonstruktion und Aneignung bewegen (6), und schließlich in einer Schlussbemerkung nochmals auf die allgemeine Frage nach der Ausbildung und Transformation invektiver Gattungen zurückkommen (7).

\section{Was sind Wutreden?}

Im zeitgenössischen Sprachgebrauch werden als Wutreden typischerweise öffentliche Reden bezeichnet, meist von Funktionsträgern wie Trainern oder auch Politikern (und übrigens kaum je von Trainerinnen oder Politikerinnen), in denen diese in emotionaler Weise und meist spontanimpulsiv Kritik üben. Diese Kritik kann an das anwesende Publikum oder auch an abwesende Dritte adressiert sein. Sie richtet sich aber immer gegen Personen und nicht etwa gegen Strukturen oder Artefakte und hat nicht zuletzt wegen der meist derben Stillage invektives Potenzial.

Das Wort ,Wutrede' wurde von Redakteur*innen der BILD-Zeitung in der Berichterstattung über Giovanni Trapattonis bereits erwähnte Pressekonferenz im Jahr 1998 geprägt, in der der italienische Trainer die Spieler der von ihm betreuten Mannschaft für mangelnde Disziplin öffentlich gerügt hatte. Das Wort blieb für die ersten Jahre gleichsam metonymisch an genau dieses Redeereignis gebunden, bis im Jahr 2003 der damalige Fußballbundestrainer Rudi Völler in 
einem berühmt gewordenen spielanschließenden Interview die TV-Experten Günther Netzer und Gerd Delling heftig angriff. Auch dieses Interview wurde in vielen Zeitungen als ,Wutrede' bezeichnet. Seitdem nimmt die Verwendung des Wortes in deutschsprachigen Zeitungen stetig zu, wie sich etwa in einer Recherche in den Pressearchiven des Deutschen Referenzkorpus (DeReKo) zeigen lässt. ${ }^{7}$ Eine Auswertung der Belege nach Themen ${ }^{8}$ zeigt zudem, dass bis heute die meisten als Wutreden klassifizierten Reden im Bereich des Fußballs gehalten werden, aber auch in den Bereichen der Politik und der Kunst wird immer wieder über Wutreden berichtet. Ein vielbeachtetes Beispiel war etwa die Reaktion des Politikers Christian Lindner auf einen Zwischenruf im Parlament, die anschließend in verschiedenen OnlineNachrichtenportalen und sozialen Medien weiterverbreitet wurde. Und tatsächlich hat sich der Diskurs über Wutreden weitgehend ins Internet verlagert, wo die Videos der betreffenden Redeereignisse in Online-Artikel oder Social Media Posts eingebettet werden können und nicht selten zu regelrechten YouTube-Hits werden.

Betrachtet man nun die als Wutreden bezeichneten Redeereignisse genauer, fällt auf, dass damit mitnichten nur Reden im engeren Sinne des Wortes, also zu einem bestimmten Anlass vorbereitete und vor Publikum monologisch vorgetragene Reden bezeichnet werden. Schon das für die Wutrede prototypische Setting der Pressekonferenz ist durch eine grundlegende FrageAntwort-Struktur charakterisiert und mit Radiound TV-Interviews sind sogar genuin dialogische Formate vertreten. Auch heimlich mitgeschnittene Wutausbrüche, die überhaupt nicht vor gröBerem Publikum stattfanden (prominente Beispiele haben etwa der Fußballtrainer Claus-Dieter Wollitz und der Politiker Winfried Kretschmann geliefert), wurden als ,Wutreden' verbreitet. Schon allein wegen der Vielfalt der Redeereignisse und -anlässe findet die Wutrede in klassisch rhetorischen Gattungstypologien, die etwa mit der polemischen Gattung der Invektive (oratio invectiva) durchaus vergleichbare Fälle kennt, keinen rechten Platz. Auch die für die Gattung der

7 Leibniz-Institut für Deutsche Sprache (2020) Deutsches Referenzkorpus.

8 Weiß (2005) Thematische Erschließung von Sprachkorpora.
Invektive konstitutive Herabsetzung einer öffentlichen Person ist hier nicht immer gegeben. ${ }^{9}$ Die klassische Rhetorik kennt die iracundia, den Jähzorn, der aber nur dort rhetorische Relevanz hat, wo er intentional für persuasive Zwecke nutzbar gemacht wird. ${ }^{10}$ Unter die Bezeichnung ,Wutrede' fallen indessen vornehmlich (scheinbar) spontanimpulsive Ausbrüche. Selbst in den deutlich redeaffineren Settings wie politischen Kundgebungen oder Parlamentsdebatten sind es, wie etwa bei Frank-Walter Steinmeier und Christian Lindner, meist die gerade nicht geplanten Teile wie die impulsiven Reaktionen auf Zwischenrufe, die dann aus dem Redekontext herausgegriffen und als Wutreden verbreitet werden. Wie aber lässt sich angesichts solcher Variabilität überhaupt von einer Gattung ,Wutrede' sprechen?

\section{Die Wutrede als invektive Gattung?}

Gegenüber der klassischen Rhetorik und der engen Orientierung ihrer Typologisierungen am Modellfall der vorbereiteten Rede vor Publikum sind neuere Gattungstheorien weniger strikt und fassen Gattungen als "mehr oder weniger normierte, aber historisch variable Bündelungen familienähnlicher Konstellationen von formalen und funktionalen Elementen [...], zu denen sich Einzeltexte mehr oder weniger prototypisch verhalten."11 Insbesondere in der soziologischen Gattungstheorie ist das ausbuchstabiert worden, etwa im Konzept der kommunikativen Gattungen, welches Gattungen als verfestigte Lösungen wiederkehrender kommunikativer Probleme fasst. ${ }^{12}$ Die formalen Aspekte von Gattungen, beschreibbar etwa als Ensembles lexikalischer oder phraseologischer Elemente, werden auf diese Weise funktional gedeutet und zudem über die soge-

9 Koster (1980) Invektive; Neumann (1998) Invektive. Vgl. außerdem Pausch in diesem Band.

10 Pichl (1998) Iracundia.

11 Reisigl (2014) Gattung.

12 Ayaß (2011) Kommunikative Gattungen. Eine vergleichbare Position nimmt aus literaturwissenschaftlicher Sicht Voßkamp ein, wenn er Gattungen als sinnstiftende Konstellationen definiert, „in denen [...] bestimmte historische Problemstellungen bzw. Problemlösungen oder gesellschaftliche Widersprüche artikuliert und aufbewahrt sind." Voßkamp (1977) Gattungen, S. 32. 
nannte Außenstruktur an soziale Kontexte angebunden. Eine wichtige Implikation dieses Ansatzes ist, dass Gattungsunterscheidungen nicht rein wissenschaftliche ex post-Unterscheidungen sind. Sie sind Teil des prozeduralen Wissens der Kommunizierenden und für diese selbst handlungsrelevant, was sich insbesondere in den von innen verwendeten Gattungsbezeichnungen niederschlägt, die immer auch eine ordnende Funktion haben. ${ }^{13}$ Aus dieser Perspektive ist schon die Gebräuchlichkeit des Wortes ,Wutrede' Anlass genug, die Gattungsmerkmale von Wutreden zu bestimmen und präziser noch zu fragen, inwiefern die Wutrede als eine invektive Gattung beschrieben werden kann.

Auf der Grundlage eines Datenkorpus von insgesamt 30 Wutreden (d.h. Videomit- und -zusammenschnitten von Reden, die in der Berichterstattung als solche bezeichnet wurden) im Umfang von 2:10 Stunden und in transkribierter Form von rund 20.000 Wörtern können folgende Merkmale einer prototypischen Wutrede herausgearbeitet werden, wobei der Prototypik entsprechend einzelne Exemplare mehr oder weniger stark davon abweichen können: ${ }^{14}$ Sie wird in einer öffentlichen Situation gehalten und ist typischerweise auch an ein öffentliches Publikum adressiert. Die Teilnehmendenkonstellation entspricht den Strukturen massenmedialer Kommunikation mit der typischen Mehrfachadressierung, die Redner*innen müssen sich also des erweiterten Kreises von Rezipierenden auch jenseits des unmittelbar anwesenden Publikums gewahr sein. ${ }^{15}$ Die Wutreden sind typischerweise responsiv orientiert, sie sind meist eine Reaktion auf Kritik, die als unangemessen und herabsetzend empfunden wird und deshalb Anlass gibt, die Produzierenden oder auch nur Überbringenden dieser Kritik ihrerseits zu kritisieren und herabzusetzen. ${ }^{16}$ So moniert Rudi Völler in Richtung der TV-Experten Netzer und Delling "diesen Scheiß, der da immer gelabert wird", und der damalige Manager des

13 Miller (1984) Genre as social action, S. 155.

14 Meier (2016) Wutreden, S. 44-51.

15 Burger/Luginbühl (2014) Mediensprache, S. 21.

16 Dies erinnert an die auf Aristoteles zurückgehende und die europäische Literaturgeschichte grundlegend prägende Theorie des Zorns (orgé) als „ein mit Schmerz verbundenes Streben nach einer vermeintlichen Vergeltung [...] für eine vermeintliche Herabsetzung" Aristoteles (2002), Rhetorik, Abs. 1978a. Vgl. Lehmann (2019) Zorn und Wut, S. 180.
FC Bayern München ruft in der Jahreshauptversammlung den sich kommerzialisierungskritisch positionierenden Fans entgegen: "Was glaubt ihr eigentlich wer ihr seid? Es kann doch nicht sein, dass wir hier kritisiert werden, dafür dass wir uns seit vielen Jahren den Arsch aufreißen." Ganz im Sinne des Konzepts der Invektivität sind Wutreden also oft Teil "kommunikative[r] Kaskaden wechselseitiger invektiver Adressierung, von Anschlusskommunikationen, die den invektiven Charakter einer Äußerung rekursiv aufnehmen, verstärken oder zuallererst erzeugen." ${ }^{17}$

In der Interaktionslinguistik, die ganz in diesem Sinne von der grundlegenden Interaktivität und Prozessualität von mündlicher Kommunikation ausgeht, ${ }^{18}$ sind typische Merkmale emotionsund besonders wutgeladener Interaktion etwa in Streitkontexten untersucht worden, die sich auch in den Wutreden wiederfinden. ${ }^{19}$ Die in den eben zitierten Ausschnitten erkennbare vulgäre Lexik, aber auch Schimpfwörter wie "Deppen" und andere herabsetzende Formulierungen wie "der hat von Tuten und Blasen keine Ahnung" gehören ebenso zum typischen Inventar der Wutreden wie die proximalen Anredeformen ("das weißt du Waldi") oder dialektale Ausdrücke. Sie alle haben gemein, dass sie nicht das in den offiziellen Redekontexten eigentlich erwartbare Register darstellen und den von den üblichen Routinen abweichenden Status der Wutreden deutlich markieren. Dabei wird dieser exponierte Status auch von den Rezipierenden thematisiert, wenn es etwa in den Kommentaren zum Facebook-Post der Zeitung Die Welt zur ,Wutrede' von Christian Lindner heißt: „Endlich mal jemand der den Nagel auf den Kopf trifft und das Ausspricht was sich viele nicht getrauen." 20 Statt der üblichen Routinen des parlamentarischen Alltags sind es gerade diese impulsiven Momente, die von den Nutzenden der Sozialen Medien begeistert aufgegriffen werden.

Eine interessante Eigenart von Wutreden, die abermals auf ihren responsiven Charakter verweist, sind auch die vielen direkten Redewiedergaben gerade der als unangemessen dargestell-

17 Ellerbrock/Koch/Müller-Mall et al. (2017) Invektivität, S. 13. Vgl. hierzu auch Pausch in diesem Band.

18 Deppermann (2008) Gespräche analysieren, S. 8 f. 19 Spiegel (2011) Streit, S. $231 \mathrm{f}$.

20 https://www.latest.facebook.com/WELTnext/videos/ 788263411221273/ (letzter Zugriff: 18.11.2020). Die Schreibung wurde unbereinigt übernommen. 
ten Kritik von anderen. Die Redewiedergaben selbst sind meist prosodisch auffällig markiert, überzeichnen so die wiedergegebenen Sprechenden und geben sie ihrerseits der Lächerlichkeit preis. Günthner weist in ihrer Analyse von Redewiedergaben im Gespräch darauf hin, dass solche Stilisierungen "die Rezipierenden zur gemeinsamen Verurteilung des porträtierten Verhaltens ein[laden]". ${ }^{21}$ Sie dienen also der "Formierung emotionaler Gemeinschaften"22 und fungieren durch die gemeinsame Herabsetzung als interaktive Ressource der Vergemeinschaftung.

Der emotional aufgeladene Charakter der Wutreden zeigt sich auch an anderen oberflächensprachlichen Merkmalen. Prosodisch fällt zuvorderst die oft erhöhte Lautstärke auf, aber auch das von Fiehler beschriebene "insistierende Iterieren"23 wie etwa in der Wutrede des Fußballtrainers Thomas Doll: "das ist doch alles bla bla bla ist das doch - alles bla bla bla ist das". Neben diesen sprachlichen Phänomenen finden sich natürlich auch gestische und mimische Auffälligkeiten, etwa heftiges und ausladendes Gestikulieren oder ein Gesichtsausdruck, der in der Forschung oft als "anger face" 24 beschrieben wird.

Obwohl sich Wutreden in dieser Weise als Bündelungen inhaltlicher, formaler und auch funktionaler Merkmale beschreiben lassen, führen derartige Beschreibungsansätze in diesem besonderen Fall nicht weiter. Das liegt weniger daran, dass nur ein Teil der Wutreden die beschriebenen Merkmale aufweist, zumal diese abgestufte Relevanz der Gattungsmerkmale durch die angenommene prototypische Struktur der Gattungen schon aufgefangen wird. ${ }^{25}$ Schwerer wiegt der Einwand, dass das zentrale Bestimmungsmerkmal kommunikativer Gattungen, historisch verfestigte Lösungen wiederkehrender kommunikativer Probleme zu sein, die als vorgefertigte Muster zur Verfügung stehen, hier kaum angewendet werden kann. Der spontan-impulsive Charakter der meisten Wutreden versperrt sich einer solchen Beschreibung, auch wenn bei medienerprobten Figuren wie Fußballtrainern fraglich ist, ob in dem

21 Günthner (2002) Stimmenvielfalt im Diskurs, S. 66. 22 Ellerbrock/Koch/Müller-Mall et al. (2017) Invektivität, S. 10.

23 Fiehler (1993) Grenzfälle des Argumentierens, S. 161.

24 Sell (2019) The Evolutionary Psychology of Anger, S. 179.

25 Günthner/Knoblauch (1994) Forms are the food of faith, S. 705. medialen Kommunikationssetting überhaupt von spontan-impulsiver Kommunikation ausgegangen werden kann. Auch nach Greta Thunbergs Auftritt vor den Vereinten Nationen ist kontrovers diskutiert worden, ob die prosodisch und mimisch zum Ausdruck gebrachten Emotionen eine geplante Inszenierung oder aufrichtiger Emotionsausdruck waren. Vor allem aber werden Wutreden erst in der Rezeption zu Wutreden gemacht, nicht selten zur Überraschung oder gar gegen den Willen der Wutredner*innen selbst. 'Wutrede' ist - vergleichbar etwa mit dem ,Skandalauftritt' - in erster Linie eine rekonstruktive Kategorie, die einen stärker rezeptionsorientierten Gattungsbegriff erforderlich macht. Zur Bestimmung der Wutrede als Gattung genügt es nicht, die ohnehin höchst disparaten Redeereignisse in den Blick zu nehmen. Vielmehr müssen auch die Interpretationsleistungen der Rezipierenden Berücksichtigung finden, wie sie in metapragmatischen Rahmungen zum Ausdruck kommen und hier auch empirisch beobachtbar sind.

\section{Doing genre}

Ein solcher rezeptionsorientierter Gattungsbegriff ist etwa in der Rezeptionsästhetik mit ihrem Begriff des Erwartungshorizontes ${ }^{26}$ schon angedeutet und dann vor allem in der amerikanischen Linguistischen Anthropologie ausformuliert worden. Hanks zufolge sind Gattungen weniger als formal bestimmbare Gruppen inhaltlicher oder stilistischer Merkmale anzusehen denn als Orientierungsrahmen und interpretative Prozeduren, die nicht zu den Texten selbst gehören, sondern beschreiben, wie sich Akteure zu Texten verhalten. ${ }^{27}$ Briggs und Bauman argumentieren in ähnliche Richtung, dass insbesondere publikumsseitige Konstruktionen intertextueller Bezüge und die hierdurch eingebrachten Deutungsrahmen die Gattungshaftigkeit von Texten ausmachen und nicht so sehr die formalen Eigenschaften der Texte selbst. ${ }^{28}$

In Anbetracht der Tatsache, dass Wutreden vor allem über digitale und Soziale Medien wei-

26 Jauß (1977) Theorie der Gattungen, S. 330.

27 Hanks (1987) Discourse genres.

28 Briggs/Bauman (1992) Genre, Intertextuality, and Social Power, S. 147. 
terverbreitet werden, ist ein solcher rezeptionsorientierte Gattungsbegriff besonders attraktiv. In diesen medialen Settings lassen sich vielfältige Praktiken der Neueinbettung, der Rekombination und der Rekontextualisierung von Diskursfragmenten beobachten, welche die ursprünglichen kommunikativen Zwecke dieser Fragmente tendenziell suspendieren. ${ }^{29}$ Auf die Wutreden angewendet zeigt sich, dass Wahlkampfauftritte, Telefon-Interviews, Dankesreden und Pressekonferenzen in der Online-Berichterstattung und den Timelines der Sozialen Medien aus ihrem ursprünglichen Kontext herausgelöst und neu eingebettet werden. Und eben durch die Bezeichnung als Wutrede werden sie metapragmatisch gerahmt und auch interpretativ vorstrukturiert. Die Gattungszuordnungen, die auf dieser Ebene der Rekontextualisierung vorgenommen werden, sind entscheidend.

Eigens für den Anwendungsfall der Sozialen Medien hat Lomborg einen Gattungsbegriff vorgeschlagen, der ganz in diesem Sinne die metadiskursiven Bezugnahmen der Kommunizierenden und die hierdurch an die eigentlichen Texte herangetragenen Normen in den Fokus rückt:

[...] genre can be studied and analysed within an interactionist framework focusing on how users 'do' genre - that is, on how users accomplish meaningful communication by bringing interactional norms, conventions and tacit genre knowledge into play in the communicative process, and on how they demonstrate genre skills. ${ }^{30}$

Die Formulierung „how users ,do' genre" erinnert an die ethnomethodologische Doing-Terminologie, wie sie etwa im gendertheoretischen Ansatz des Doing Gender Verwendung findet. In diesem Ansatz wird das soziale Geschlecht nicht als substantielle Eigenschaft von Personen aufgefasst, sondern als ein "routine accomplishment", das Geschlechterunterschiede in der Interaktion relevant setzt und dadurch Geschlecht erst diskursiv konstruiert. ${ }^{31}$ In Anlehnung hieran schlagen Meier und Marx für gattungstheoretische Fragen das

29 Meier/Viehhauser (2020) Rekontextualisierung, S. 6f.; Jones (2018) Surveillant media, S. 252; Krieger/Machnyk (2019) Das Internet ist für alle Neuland.

30 Lomborg (2011) Social Media, S. 68.

31 West/Zimmerman (1987) Doing Gender, S. 125; Goffman (1977) The Arrangement between the Sexes, S. 324. Vgl. hierzu auch Kanzler (in diesem Band), die ebenfalls
Konzept des Doing Genre vor. ${ }^{32}$ Gattungsunterscheidungen und -zuordnungen, die im Reden über Wutreden faktisch vorgenommen werden, werden nicht als Ausdruck tatsächlicher substantieller Eigenschaften der betreffenden Redeereignisse aufgefasst, sondern als Orientierungsmuster, die im - meist nachträglichen - Reden über Wutreden erst entstehen. So rücken metadiskursive Praktiken in den Fokus, welche auf vielfältige Weise die Gattungshaftigkeit der Wutreden indizieren oder auch thematisieren.

Empirische Belege für solche Praktiken des Doing Genre finden sich im Falle der Wutreden etwa in den Überschriften der Online-Artikel, in denen über die Wutreden mit oftmals direkt eingebetteten Videos berichtet wird. "Wutrede von Steinmeier wird zum Hit bei YouTube" heißt es etwa in einem Bericht über einen Wahlkampfauftritt des damaligen Außenministers ${ }^{33}$ und ein Video einer AfD-kritischen Rede Cem Özdemirs wird mit der Schlagzeile "Özdemirs Wutrede sorgt für Begeisterung" weiterverbreitet. ${ }^{34}$ Entscheidend hierbei ist, dass es erst die - häufig zusammengeschnittenen - und bereits in den Sozialen Medien verbreiteten Videos, und weniger die in innen abgebildeten Redeereignisse selbst, die Diskursfragmente sind, die dann mit dem Label ,Wutrede' versehen werden. Nicht zuletzt durch den Verweis auf ihre Viralität werden sie auch interpretativ vorstrukturiert und letztlich als Unterhaltungsprodukte präsentiert. In konsequenter Fortführung dieses Unterhaltungscharakters finden sich auch zahlreiche Listicles, ${ }^{35}$ also Kompilationen wie "Die besten Wutreden" oder "Die legendärsten Wutreden", in denen die Videos meist gleich eingebettet sind. ${ }^{36}$ Interessanterweise werden hier auch längst vergangene Redeereignisse wie der Wutausbruch Klaus Kinskis am Set des Films Fitzcarraldo oder die Parlamentsreden Herbert Wehners rückwirkend als Wutreden umgedeutet und gerade in ihrer seriellen Präsentation mit anderen Wutreden zu Gattungsexemplaren erhoben. Es werden also intertextuelle Bezüge

den Zugang zu Genres und Genredefinitionen über textinhärente Eigenschaften (properties) kritisch sieht.

32 Meier/Marx (2019) Doing genre.

33 Bitz (2014) Wutrede von Steinmeier wird zum Hit bei YouTube.

34 Thomys (2018) Özdemirs Wutrede sorgt für Begeisterung.

35 Pflaeging (2020) Diachronic perspectives.

36 Brauer (2015) Wutredner und Choleriker. 
hergestellt, welche die ursprüngliche, situationsgebundene Funktionalität der Redeereignisse völlig suspendieren und mit Epitheta wie ,schön', ,begeisternd', ,episch', ,legendär' usw. ganz neue Beschreibungsmaßstäbe etablieren, die erst auf der metadiskursiven Ebene Geltung haben.

Im Übrigen leistet schon die Bezeichnung ,Wutrede' selbst durch ihre Semantik einen Beitrag zur metapragmatischen Rahmung, indem durch das Zweitglied, -rede' auch bei eigentlich dialogischen Settings vor allem der monologisch-performative Charakter der Redeereignisse hervorgehoben und geradezu ein staging der affektiv aufgeladenen Kommunikation suggeriert wird. Wutreden sind zwar durchaus tendenziell monologisch und suspendieren vorübergehend die üblichen Mechanismen des Sprecherwechsels. Ein eindrückliches Beispiel ist etwa eine nur 42-sekündige Pressekonferenz des Fußballtrainers Klaus Augenthaler, in der dieser die Fragen selbst gestellt und auch selbst beantwortet hat. ${ }^{37}$ Doch auch bei den eher dialogischen Varianten wie etwa dem spielanschließenden TV-Interview von Rudi Völler rückt die Bezeichnung ,Wutrede' die gesprächsförmigen Aspekte in den Hintergrund, sodass auch nur über Völlers Redebeiträge in den Medien berichtet wurde. Auch das Erstglied ,Wut-' hat einen unifizierenden Effekt. Gleichwohl man aus emotionstheoretisch fundierter Sicht die in den Wutreden zum Ausdruck gebrachten Emotionen viel präziser unterscheiden müsste - Greta Thunbergs Ansprache vor den Vereinten Nationen etwa ist wohl eher durch die moralisch aufgeladene Emotion der Verachtung charakterisiert ${ }^{38}$-, wird alles kurzerhand der Wut zugerechnet. Wie auch die häufigen Paraphrasen wie ,Ausraster' zusätzlich verdeutlichen, werden die betreffenden Redeereignisse schon dadurch als spontaneruptiv gerahmt. Die faktischen Unterschiede in den Motivationslagen der Redner*innen rücken in den Hintergrund.

Eine weitere Praktik des Doing Genre, die auf ein gewisses Gattungsbewusstsein hinweist und dieses auch diskursiv formiert, bilden die häufigen vergleichenden Bezugnahmen insbesondere auf die traditionsstiftende Wutrede von Trapat-

37 https://www.welt.de/sport/article864475/Was-erlauben-Klaus-Augenthaler.html (letzter Zugriff: 18.11.2020).

38 Brokoff/Walter-Jochum (2019) Verachtung und Hass aus literaturwissenschaftlicher Sicht. toni. "Heißsporn Gattuso macht den Trapattoni" heißt es etwa in einem Bericht über die Wutrede des italienischen Trainers. ${ }^{39}$ Vor allem aber in den Kommentarbereichen der Sozialen Medien, wo die Wutredenvideos kursieren, finden sich geradezu Aushandlungen von Gattungsnormen: ${ }^{40}$ "Wut? Also eine emotionale Wutrede sieht für mich anders aus, siehe Erdogan."41 Durch solche Kommentare, die interessanterweise durch die Formulierung ,sieht für mich ... aus' den Rezeptionsprozess selbst fokussieren, explizieren die Rezipierenden ihre Erwartungshorizonte, die aus ihrer Perspektive die Gattung der Wutrede formieren. ${ }^{42}$

Man muss also zwischen den ursprünglichen Redeereignissen selbst und den medial rekontextualisierten, meist serialisiert präsentierten und damit auch metapragmatisch neu gerahmten Aufzeichnungen dieser Redeereignisse unterscheiden. Erst auf dieser zweiten Stufe werden sie aktiv in einen Gattungszusammenhang gestellt, und nur in Bezug auf diese medial rekontextualisierten Ereignisse ergibt die Rede von Wutreden als Gattung überhaupt Sinn. Das hat der amerikanische Blogger Travis Timmons im Übrigen klar erkannt, wenn er in einem Artikel "Wutrede Hall of Fame: Giovanni Trapattoni, the Original"43 seinen amerikanischen Leser*innen das in seinen Augen typisch deutsche Phänomen der Wutrede (er lässt den Ausdruck unübersetzt) erklärt. Die Wutrede definiert er als "an event captured on camera, featuring an enraged male", und gerade die mediale Repräsentation, insbesondere auf YouTube, und damit auch das veränderte Publikum transformieren den pragmatischen Gehalt der ursprünglichen Redeereignisse grundlegend: „From an audience perspective, it seems we've mostly hijacked Wutreden with irony, transforming them into legendarily humorous episodes." Nach dieser Charakterisierung präsentiert er Trapattonis Wutrede als "THE Wutrede. The ,O.G.' of Wutreden. The godfather", also als das prototypische Gattungsexemplar, und bettet auch das entsprechende YouTube-

39 o.V. (2014) Heißsporn Gattuso macht den Trappatoni. 40 Lomborg (2011) Social Media, S. 62.

41 Die Äußerung war im inzwischen deaktivierten Kommentarbereich zu diesem Artikel zu finden: https://www. spiegel.de/politik/ausland/steinmeier-bruellt-montagsdemo-auf-alexanderplatz-in-berlin-nieder-a-970571.html (letzter Zugriff: 18.11.2020).

42 Hiippala/Tseng (2017) Media evolution.

43 Timmons (2018) Wutrede Hall of Fame. 
Video ein. Damit fängt der Blogger zum einen die Spezifika von Wutreden als medial rekontextualisierte und metadiskursiv neu gerahmte Redeereignisse präzise ein und liefert zum anderen auch selbst ein besonders dichtes Beispiel für das Doing Genre von Wutreden.

Der gattungsanalytische Perspektivenwechsel von den ursprünglichen Redeereignissen hin zu ihren medialen Rekontextualisierungen liefert dann auch Erklärungsmöglichkeiten für den eingangs beschriebenen Anstieg in der Häufigkeit von Wutreden. Öffentliche Wutausbrüche als solche sind ja mitnichten ein neues Phänomen. Aber das Internet und insbesondere die Sozialen Medien schaffen mit ihren Affordanzen der, persistence', ,spreadability' und ,visibility ${ }^{144}$ neue Rezeptionsbedingungen, welche die gattungskonstitutiven Rekontextualisierungen erleichtern, den Praktiken des Doing Genre Raum geben und auch die nötige Sichtbarkeit verschaffen. Die Wutreden geben somit ein eindrückliches Beispiel dafür $a b$, wie eine invektive Praxis - denn die Wutreden haben als Form der Kritik ad personam ja durchaus herabsetzendes Potenzial - metadiskursiv umgedeutet werden kann und wie gerade Gattungszuordnungen diskursive Ordnung schaffen können. Die aus dem Invektiv-Geschehen resultierenden Effekte, so heißt es im Konzeptpapier zum Begriff der Invektivität, "häng[en] von den vielfältigen Möglichkeiten der Deutung und den entsprechenden Resonanzen ab, die es selbst erst erzeugt. " 45 Wie vielfältig diese Deutungsmöglichkeiten sein können und wie sie sich (inter-)medial ausgestalten, lässt sich am Beispiel der Wutreden empirisch detailliert zeigen. Und wie weitreichend die Effekte sind, zeigt sich in neueren Entwicklungen, die darauf hindeuten, dass sich die Wutrede von einer bloß rekonstruktiven zu einer nunmehr auch produktiven Kategorie zu wandeln beginnt.

\section{Von der Rezeption zur Aneignung}

Das Gattungswissen über die Wutreden, wie es sich in den beschriebenen Praktiken des Doing Genre

44 Boyd (2014) It's complicated, S. 11.

45 Ellerbrock/Koch/Müller-Mall et al. (2017) Invektivität, S. 6. diskursiv manifestiert, betrifft zunächst und vor allem die nachträgliche Kategorisierung, Benennung und mithin auch Deutung der betreffenden Redeereignisse. Gerade in jüngerer Zeit lassen sich aber Tendenzen beobachten, dass das Gattungswissen selbst produktiv wird. War ,Wutrede' zunächst eine Fremdkategorisierung, welche im Übrigen die Wutredner*innen nicht selten einem voyeuristischen Blickregime unterwirft und die Aufmerksamkeit mitunter auf körperliche Merkmale wie schrille Stimmen, rot angelaufene Gesichter oder geschwollene Halsschlagadern lenkt, eignen sich Akteure diese Kategorie zusehend gezielt an. Das deutet sich schon in den Fällen an, in denen Pressetexte, die man in klassischer Gattungsterminologie wohl als polemische Glossen bezeichnen könnte, von den Zeitungen selbst als Wutreden präsentiert werden. ${ }^{46}$ Wenigstens in einem spielerischen Rahmen können die als typisch wahrgenommenen Merkmale von Wutreden aber auch direkt handlungsleitend werden. So präsentiert das Fußballmagazin 11Freunde eine als Lückentext gestaltete Anleitung für die "perfekte Wutrede”, die für den persönlichen Gebrauch ausgestaltet werden kann:

Jetzt möchte ich mal in aller Deutlichkeit was sagen: Mir reicht es! Wirklich! Wenn ich den Namen ___ (hier bitte den Namen eines Ex-Spielers einfügen) schon höre, kommt mir das Frühstück wieder hoch. Das ist schade um das Omelett, aber es geht nicht anders. Wer hat denn hier in den letzten Jahren den Ruf des Klubs gerettet? War das (hier bitte den Namen des Ex-Spielers einfügen)? So ein Schwachsinn! ${ }^{47}$

Auch paraverbale sowie gestische und mimische Anweisungen wie etwa Schnaufen vor Wut, krachend auf den Tisch hauen und ein starrer Blick finden sich in dem Text, der deutlich macht, wie sehr sich die Gattungserwartungen an Wutreden bereits konsolidiert haben. Interessanterweise rückt gerade in dieser fingierten Wutrede, die als besonders verdichtetes Beispiel gesehen werden kann, vor allem ihr invektiver Charakter, die persönlich herabsetzende und affektiv grundierte Kritik ad hominem, in den Vordergrund.

Auf YouTube und Facebook finden sich aber neuerdings auch Fälle, in denen einzelne Nutzer*innen ganz gezielt auch ausdrücklich als solche bezeich-

46 https://twitter.com/szmagazin/status/9779780314815 40608 (letzter Zugriff: 18.11.2020).

47 Kuhlhoff (2017) „Da kotze ich doch im Strahl” - Wie sieht die perfekte Wutrede aus? 
nete Wutreden produzieren, als geplante und für die Videokanalabonnenten inszenierte Wutausbrüche und Schimpftiraden. So beschimpft ein Fan den Trainer des von inm favorisierten Vereins als "Absturztrainer" und "Witz-Lauch-Typen", der so "dumm, behindert, gehirnamputiert" sei, dass er "wenn überhaupt" für die „Bezirksliga" geeignet sei. ${ }^{48}$ Der HSV-Fan und selbsterklärte "Direkt-Ansager" Timo Siever publiziert auf seiner Facebook-Seite regelmäßig als Wutreden bezeichnete Selfie-Videos von sich in seinem Auto, in denen er das eigene Team nach schlechten Spielen als "nicht ganz dicht" oder auch Ultra-Fans des eigenen Vereins als "Schwachmaten" beschimpft, da sie mit ihren „Bengalos" für hohe Geldstrafen verantwortlich sind. ${ }^{49}$ Die an die Auftritte der Kunstfigur Gernot Hassknecht in der Comedy-Show heute-show erinnernden Videos, die meist in ruhigem Ton beginnen, um dann schlagartig in lautes Schreien umzuschlagen, werden auch in Online-Medien verschiedentlich aufgegriffen und ihrer offenkundigen Inszeniertheit zum Trotz als authentische Ausraster eines enttäuschten Fans weiterverbreitet. ${ }^{50}$

Besonders aufschlussreich ist in diesem Zusammenhang auch ein selbstproduziertes YouTube-Video der ehemaligen Pegida-Aktivistin Tatjana Festerling mit dem Titel "Wutrede: Es geht ums Überleben - tut endlich was!". ${ }^{51}$ In dem gut siebenminütigen Video, in dessen Vorspann u.a. das Logo der rechtspopulistischen Bewegung Fortress Europe eingeblendet wird, filmt sich Festerling am Meeresufer im Gegenlicht und spricht direkt in die Kamera (auffällige Betonungen sind im Transkript mit Unterstreichungen markiert):

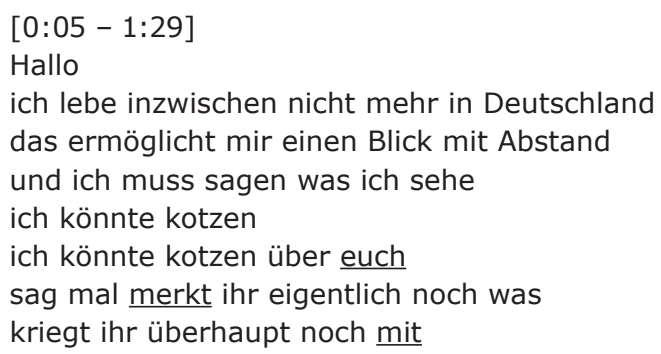

48 https://www.youtube.com/watch?v=OW-Ey36MsoM (letzter Zugriff: 18.11.2020).

49 https://www.facebook.com/DerSievi/ (letzter Zugriff: 18.11.2020).

50 https://www.youtube.com/watch?v=e68-oPtCBfE (letzter Zugriff: 18.11.2020).

51 https://www.youtube.com/watch?v=bOigXb_9L28 (letzter Zugriff: 18.11.2020). empfindet ihr noch etwas zu dem was bei rundherum um euch passiert

oder versucht inr das alles nur noch irgendwie mit dem Verstand zu abstrahieren

und euch an irgendwelche komischen schalen Illusionen an irgendwelche Gedanken zu klammern

wird schon nichts passieren

es is noch immer jot jejangen

oder ach der Kelch geht schon an uns vorbei

$\mathrm{n} \underline{\mathrm{Schei} ß}$ wird er

gar nichts geht an euch vorbei

Himmelherrgott fangt endlich an wirklich erwachen erwacht endlich

spürt mal was bei euch los ist

spürt mal die Verzweiflung die Hoffnungslosigkeit

und fangt nicht immer an im Verstand alles irgendwie zu rationalisieren

Bullshit Schwachsinn

Polizisten werden stranguliert

Frauen sollen nicht mehr alleine joggen gehen

was ist denn das bitteschön für ein Land

ist doch $\mathrm{n}$ lach lachhaft

weil Kinder werden in den Schulen drangsaliert zusammengeschlagen abgezogen

ja wir schützen noch nicht mal unsere Kinder was ist denn das für ne beschissene Gesellschaft die sich nicht mehr um ihre Nachkommen kümmert $[\ldots]$

Bemerkenswert an dieser ,Wutrede' ist die normativ unterlegte und durch den ganzen Auftritt einschließlich der Mimik auch performativ inszenierte Gegenüberstellung von ,Verstand' und ,Gefühl', von Rationalisierungsversuchen und dem Spüren der Verzweiflung - eine Gegenüberstellung, die in deutlicher Parallele zum Typus des ,Wutbürgers' dann auch die eigene Wut legitimiert und geradezu als Zustand der Erweckung erscheinen lässt. Und so ist die vorgetragene Kritik, obwohl sie in der zweiten Person Plural vorgetragen wird, wohl auch nur zum Schein an die Kanal-Abonnent*innen adressiert. Eher ist sie an die Mehrheitsgesellschaft gerichtet, welche die angeblichen Missstände in Deutschland nicht wahrnehmen will.

Rein formal finden sich Parallelen zu den herkömmlichen Wutreden, wie sie auf YouTube zirkulieren. Die insistierenden Wiederholungen, das vulgäre Vokabular, das überartikulierte Sprechen in erhöhter Lautstärke und die typische Mimik verbinden Festerlings Wutrede etwa mit der von Uli Hoeneß, sind hier aber bis ins Detail geplant. Die Wut wird ganz gezielt als solche inszeniert und, anders als bei den Fußballfans, wo der Unterhaltungscharakter im Vordergrund steht, ist die ganze Inszenierung Teil einer agitatorischen 
Strategie, die Wut zum politischen Programm macht. Ähnlich wie bei Donald Trump und seinen affektgesättigten Auftritten on- und offline wird Wut zur Leitemotion der öffentlichen Selbstinszenierung und zielt dabei auf die "Schaffung exklusiver Solidarität". ${ }^{52}$ Und gerade die indirekte Adressierung, welche die Kritik an dem ,lachhaften Schwachsinn' und den ,schalen Illusionen' an die im Video Angesprochenen zu richten scheint, tatsächlich aber die Rezipierenden als Teil einer Erregungsgemeinschaft von eben dieser Kritik ausnimmt, trägt hierzu bei und unterstreicht in ihrer rhetorischen Kunstfertigkeit den Inszenierungscharakter des Videos.

Derartige Formen der Aneignung der Kategorie ,Wutrede' zeigen, dass der Diskurs über die Wutreden die alltagsweltlich verfügbare Gattungssystematik und die entsprechenden Erwartungshorizonte schon so weit transformiert hat, dass auch Wutreden wie die von Tatjana Festerling als Medienprodukte nicht nur möglich, sondern auch erfolgreich und kommunikativ anschlussfähig sind. Die gezielte Funktionalisierung affektgrundierter Rede in den als solche inszenierten Wutreden wird dabei insbesondere in den Dienst invektiver Zwecke gestellt, die manchmal in spielerischer Rahmung eher Unterhaltungsfunktion haben, aber eben auch, wie bei Festerling, politische Dimension haben und gesellschaftliche Ein- und Ausschlüsse verhandeln können. Die Wutrede konsolidiert sich somit zu einer benennbaren und auch produktiv verfügbaren Gattung, welche auf invektive Dynamiken zurückwirkt und innen Form gibt.

\section{Ein Blick auf verwandte Fälle}

Die eben nachgezeichnete Wendung von der Rezeption hin zur Produktion über die bewusste Aneignung einer primär rekonstruktiven Kategorie wird am Beispiel der Wutrede zwar besonders deutlich, lässt sich in ähnlicher Form aber auch an anderen Fällen aufzeigen, in denen sich Formen herabsetzender Rede zu Gattungen verdichten.

Zu nennen wäre etwa die Kategorie des Shitstorms, welche kollektive und allein schon deshalb höchst disparate Diskursereignisse rekonst-

52 Koch/Nanz/Rogers (2020) The Great Disruptor, S. 7. ruktiv ordnet. Das Neologismenwörterbuch OWID definiert Shitstorm als "unkontrollierte[n] virtuelle[n] Sturm der Entrüstung als Reaktion auf die Äußerung einer bekannten Person in Form von massenweise versendeten beleidigenden und bedrohlichen E-Mails oder Facebook-Nachrichten, der von den Medien aufgegriffen wird". ${ }^{53}$ Gerade die abschließende Einschränkung macht deutlich, dass die Medienresonanz und die benennende Kategorisierung als Shitstorm diesem Diskursphänomen wesentlich sind. Typischerweise werden in der Berichterstattung einzelne Tweets oder Facebook-Kommentare herausgegriffen und verdichtend so rekontextualisiert, dass die Kategorisierung als Shitstorm gerechtfertigt erscheint, auch wenn im tatsächlichen Diskurs die Shitpostings womöglich in der Minderheit sind. ${ }^{54}$ Als gattungstypologische Kategorie wird der Shitstorm also auch durch (inter-)mediale Praktiken des Doing Genre konturiert.

Neuere Entwicklungen zeigen nun, dass sich Akteure diese retrospektive und typischerweise anklagende Kategorisierung selbst zu eigen machen können. So wird das Verb shitstormen nunmehr auch als fremdes wie eigenes Verhalten bezeichnendes Kommunikationsverb verwendet: „wenn ich shitstormen oder mich aufregen/ ärgern würde...dann sähe das ganz anders aus." 55 Und auch parodistische Anleitungen für gelungenes Shitstormen finden sich inzwischen im Netz. ${ }^{56}$

Eine andere Form der Aneignung zeigt sich dagegen in einem Facebook-Post des GrünenPolitikers Boris Palmer, in dem er einen Screenshot der Startseite der Deutschen Bahn, auf dem Fahrgäste verschiedener ethnischer Hintergründe abgebildet sind, postet und mit folgendem Kommentar versieht:

Der shitstorm wird nicht vermeidbar sein. Und dennoch: Ich finde es nicht nachvollziehbar, nach

53 https://www.owid.de/artikel/402347 (letzter Zugriff: 18.11.2020).

54 Meier/Marx (2019) Doing genre, S. 205; Marx (2019) Von Schafen im Wolfspelz, S. 146.

55 http://swtorcantina.de/paketoeffnen-neu-gestaltet/ (letzter Zugriff: 18.11.2020); Dieser und weitere Belege lassen sich im Webkorpus 2016c im Digitalen Wörterbuch der Deutschen Sprache dwds.de recherchieren: https:// www.dwds.de/r?q=shitstormen\&corpus=ibk_web_2016c (letzter Zugriff: 18.11.2020).

56 Gogl/Lebersorger (2016) Shitstormen auf Österreichisch \#oidaRP. 
welchen Kriterien die "Deutsche Bahn" die Personen auf der Eingangsseite ausgewählt hat. Welche Gesellschaft soll das abbilden ${ }^{57}$

Durch seine abschließende Frage scheint Palmer zu insinuieren, dass die Bebilderung der DBHomepage einer identitätspolitischen Agenda folge, die in einer Überbetonung von Multikulturalität den eigentlichen ethnischen Charakter der deutschen Gesellschaft in den Hintergrund rückt - eine implizit rassistische Position, die so auch von rechtsextremen Akteuren wie der Identitären Bewegung vertreten wird. ${ }^{58}$ Die erwarteten Reaktionen auf diese Stellungnahme kategorisiert Palmer nun vorgreifend als, Shitstorm' und delegitimiert sie somit als problematische, einer unheilvollen Entrüstungslogik folgende Praxis. In typisch rechtspopulistischer Manier inszeniert sich Palmer als Tabubrecher, der sich dem Diktat der politischen Korrektheit nicht beugt, und verschiebt so die Aufmerksamkeit weg von der eigenen rassistischen Äußerung hin zu der vermeintlichen Überreaktion des Publikums. ${ }^{59}$ Diese Reaktionen aber werden durch ihre Kategorisierung als ,Shitstorm' in einen vereinheitlichenden Deutungsrahmen gestellt. Das zunächst vorgreifende Doing Genre setzt Palmer dann übrigens parallel zu den tatsächlich einsetzenden Reaktionen fort, wenn er nach etwa einer Stunde seinen Facebook-Post ergänzt:

PS: Eine Stunde später tobt der Shitstorm. Wie vorhergesehen. Alle, die mich jetzt fragen, warum ich dieses Thema aufgreife, frage ich zurück: Wenn die Auswahl dieser Bilder vollkommen belanglos, normal, unbedeutend ist, warum regt ihr euch dann so auf? Was wir hier diskutieren, ist Identitätspolitik. Und zwar von Recht [sic!] wie Links. Die einen sagen, man wisse nicht mehr, in welchem Land man lebt, die anderen bekämpfen alte weiße Männer. Und gemeinsam haben die Identitätspolitiker es ziemlich weit damit gebracht, uns zu spalten.

Die Formulierung "tobt der Shitstorm" rahmt die in den Kommentaren vorgebrachte Kritik als ein blindes Wüten, das rationalen Argumenten kaum zugänglich ist, so wie auch die Frage "warum regt

57 https://www.facebook.com/ob.boris.palmer/ posts/2381885385184313 (letzter Zugriff: 18.11.2020).

58 Bruns/Glösel/Strobl (2017) Die Identitären; Boehnke (2019) Rechter Kulturkampf heute.

59 Niehr/Reissen-Kosch (2018) Volkes Stimme?, S. 127. inr euch dann so auf?" kritische Kommentare als unbegründet zurückweist. Nicht der eigene Rassismus erscheint hier als das Problem, sondern seine Thematisierung, welche, uns' spalte. Zwar finden sich tatsächlich zahlreiche beleidigende und verhöhnende Kommentare, wenn Palmer etwa "verbaler Brechdurchfall" attestiert wird. Dass aber viele der Kommentare sehr wohl ihre Kritik begründen, wird dabei ebenso ausgeblendet wie die vielen Kommentare, die sich um Vermittlung bemühen oder gar ausdrücklich unterstützend argumentieren. Die Kategorisierung als ,Shitstorm' hat angesichts des sehr ausdifferenzierten kommunikativen Geschehens in den Kommentaren ordnende und akzentuierende Kraft und das Doing Genre wird als rhetorisches Mittel im politischen Diskurs nutzbar.

In eine ähnliche Richtung zielt der in Political Correctness-kritischen Kontexten etablierte Begriff der ,Hate Facts' im Sinne von angeblich tabuisierten Wahrheiten, die aus der Perspektive des Mainstream-Diskurses als ,Hate Speech' kategorisiert würden. Der rechtskonservative Blog National Association of Scholars etwa definiert Hate Facts wie folgt:

Hate facts are truths that must not be spoken among the intelligentsia any more because doing so could offend some "protected" group. Academics (and anyone else who wants to survive in the public realm) learn to steer a safe course away from hate facts. ${ }^{60}$

Auch Hate Speech ist zuvorderst eine rekonstruktive Kategorie, die sich nicht zuletzt in den Sozialen Medien durch metadiskursive Thematisierungen diskriminierender Rede in den und außerhalb der digitalen Medien etabliert hat. Der Terminus ,Hate Speech' selbst ist dabei typischerweise "nicht Teil des sprachlichen Materials [...], das er kategorisiert". ${ }^{61}$ Insbesondere von rechter Seite aus wird jedoch die im Hate Speech-Diskurs oft formulierte Kritik an ehemals unhinterfragten sprachlichen Mustern der Ausgrenzung und Herabsetzung als übertriebene Political Correctness kritisiert, welche die Meinungskorridore verenge. ${ }^{62}$ Gerade weil Hate Speech zunächst eine Fremdkategorisierung ist, die jedoch für illegitim

60 Leef (2014) Hate Facts.

61 Marx (2017) Rekontextualisierung von Hate Speech, S. 132.

62 Auer (2002) Political Correctness. 
gehalten wird, können das gezielte Aussprechen von Hate Speech und der affirmative Bezug auf diese Kategorie geradezu als Akte des Widerstands erscheinen. "Ja - ich bin ein Rassist und das ist gut so!"63 formuliert in trotzigem Ton ein Kommentierender auf der rechtsextremen Internetseite pi-news und bedient zuvor etwa mit der Rede vom "Terror dieser Gutmenschen-Verbrecher" zielsicher den Topos der Meinungsdiktatur.

Wie nun gerade die Rede von Hate Facts diese vermeintliche Widerstandshaltung diskursiv umsetzt, lässt sich an der folgenden Passage aus Nils Wegners Editorial zur neurechten Zeitschrift Sezession zeigen:

\begin{abstract}
Der Autor dieser Zeilen stellt die akademischen Ikonoklasten vor, die Aktivisten gegen Multikulturalismus-, Vielfalt- und Schmelztiegellügen auf der ganzen Welt mit (wie man heute so "schön" sagt) Hate facts versorgen. Ihre wissenschaftliche Disziplin ist die Human biodiversity, kurz HBD, und ihr Anliegen ist relativ simpel: Das fortlaufende Ignorieren und Wegdiskutieren der natürlichen Unterschiede zwischen ethnischen und demographischen Gruppen befördert eine grundlegende Instabilität „bunter" Gesellschaftskonstrukte, die sich nicht dadurch aus der Welt schaffen läßt, daß man sie durch Sozialleistungen und Förderprogramme mit Geld bewirft. Ein „Haßfakt” auch das, doch nichtsdestoweniger ein Fakt. ${ }^{64}$
\end{abstract}

Die hier ganz offen vorgebrachte biologistischrassistische Anschauung einer "Human biodiversity", welche die "natürlichen Unterschiede zwischen ethnischen [...] Gruppen" verteidigt, aber auch die Verächtlichmachung von Minderheitenschutzprogrammen und ihren Subventionen werden hier als , Hate Facts' eingeordnet. Im Vorgriff auf die erwartbare Stigmatisierung solcher Positionen als Hass, die aber nur um den Preis "fortlaufende[n] Ignorieren[s] und Wegdiskutieren[s]" zu haben sei, werden sie als der Wahrheit verpflichtete Positionen in einer Welt der Lügen legitimiert. ${ }^{65}$ Die eigentlich stigmatisierende Kategorie ,Hate Speech' wird so angeeignet und schon in der Produktion verfügbar gemacht. Zugleich aber ist in die so kategorisierte Praxis auch schon ihre metadiskursive Thematisierung eingelassen.

63 http://www.pi-news.net/2013/06/video-hallo-meinname-ist-paul-weston-und-ich-bin-ein-rassist/ (letzter Zugriff: 18.11.2020).

64 Wegner (2017) Sezession, S. 79.

65 Meier (2020) Selbstlegitimationen von Hate Speech, S. 140
Ein ganzer Diskurs von Fremd- und Selbstzuschreibungen und -positionierungen im Wechsel von Kritik und Gegenkritik verdichtet sich in der Rede von ,Hate Facts'. Die "kommunikativen Kaskaden wechselseitiger invektiver Adressierung" und metainvektiver Thematisierung sowie die hierbei oft zu beobachtenden Formen "diskursive[r] Umcodierung"66 im Hate Speech-Diskurs werden auf diese Weise geordnet und auch selbst als invektive Ressource nutzbar.

\section{Schlussbemerkung}

Die in diesem Beitrag eingenommene Perspektive auf invektive Gattungen zeigt zum einen, wie die im Konzept der Invektivität so zentral gesetzten Anschlusskommunikationen und Deutungsmodi in einem diskurs- und rezeptionsorientierten Gattungsverständnis eingefangen werden können. Zum anderen veranschaulichen die besprochenen Fälle durch die prozessorientierte Perspektive auf Gattungskonstruktionen und -aneignungen auf empirisch detaillierte Weise, wie vielfältig die Deutungsmöglichkeiten von Invektiv-Geschehen sein können, wie sie sich zu Erwartungshorizonten verdichten und später auch produktive Aneignungen ermöglichen. Gerade in den digitalen und Sozialen Medien, wo Rezeptionszeugnisse verschriftlicht und dauerhaft sichtbar sind, lassen sich diese Prozesse präzise nachvollziehen, und die dort herrschenden medialen Bedingungen sind sicherlich prägend für die Ausbildung der hier diskutierten invektiven Gattungen.

Die diskutierten Beispiele repräsentieren dabei verschiedene Etappen oder auch Grade der Gattungskonstruktion. Bei der Wutrede ist sie schon weit fortgeschritten und durch den monologischen Charakter der Wutrede auch so fokussiert, dass längst gezielt neue Gattungsexemplare produziert werden können. Shitstorms als kollektiv hervorgebrachte Diskursereignisse entziehen sich dagegen der gezielten Produktion. Doch auch hier zeigen die diskutierten Möglichkeiten der vorgreifenden Kategorisierung erwartbarer Reaktionen, dass sich hier Erwartungshorizonte verdichten und die vereinheitlichende Benen-

66 Ellerbrock/Koch/Müller-Mall et al. (2017) Invektivität, S. 13. 
nung von Diskursereignissen dann auch strategisch genutzt werden kann. Hate Facts wiederum können durchaus gezielt produziert werden. Doch nicht allein das ist für den hier diskutierten Zusammenhang entscheidend, sondern vielmehr, dass diese Kategorie aus einer gezielten Aneignung der eigentlich stigmatisierenden Kategorie der Hate Speech hervorgeht und einen ordnenden Zugriff auf den Hate Speech-Diskurs erlaubt, in dem sozusagen die Fronten geklärt sind. Und mehr noch: Nicht die eigene Rede erscheint dann als hasserfüllt, sondern die Reaktion derjenigen, welche die in ihr zur Sprache gebrachten ,Fakten' angeblich nicht wahrhaben wollen.

Von den klar umrissenen und in detaillierten Gattungstaxonomien verorteten Gattungen, wie sie etwa in der Literaturwissenschaft typischerweise diskutiert werden, sind alle hier diskutierten Fälle weit entfernt. In der historisierenden Rückschau, womöglich auch vermittelt über gattungstypologische Traktate, Rhetoriken und ähnliche Schriften, mögen Gattungen wie einigermaßen stabile und formal-funktional bestimmbare Gebilde erscheinen, aus denen sich auch klare Taxonomien ableiten lassen, für die Wutreden, Shitstorms und erst recht Hate Facts viel zu unstet sind. Allerdings können gerade solche gattungstypologischen Traktate, auf die sich die Literaturgeschichte häufig beruft, als besonders wirkmächtige Formate des Doing Genre und gewissermaßen als dessen Endpunkte beschrieben werden. Die hier diskutierten Fälle können dagegen als exemplarische Beispiele für den Prozess von der zunächst rekonstruktiven Ordnung über Aneignung bis hin zur Produktion, also für die verschiedenen Verlaufsstadien des Doing Genre gesehen werden. Vielleicht wirft dies ein Licht darauf, wie man sich die Entstehung neuer bzw. Umstrukturierung bestehender Gattungen bis hin zu ihrer Verankerung im kommunikativen Haushalt insgesamt vorstellen kann.

\section{Literaturverzeichnis}

\section{Quellen}

Bitz, Xaver (2014): Wutrede von Steinmeier wird zum Hit bei YouTube. Merkur: https://www.merkur.de/politik/ wutrede-steinmeier-wird-youtube-zr-3573736.html (letzter Zugriff: 18.11.2020).
Brauer, Markus (2015): Wutredner und Choleriker: Der Ausraster - die besten Wutreden. Stuttgarter Nachrichten: https://www.stuttgarter-nachrichten. de/inhalt.wutredner-und-choleriker-der-ausrasterdie-besten-wutreden.5258caf0-ee90-47ad-b4a9a375c0dd2401.html (letzter Zugriff: 18.11.2020).

Dpa/sid/tsch (2007): Was erlauben Klaus Augenthaler? WELT: https://www.welt.de/sport/article864475/ Was-erlauben-Klaus-Augenthaler.html (letzter Zugriff: 18.11.2020).

Festerling, Tatjana: Wutrede: Es geht ums Überleben - tut endlich was! https://www.youtube.com/ watch?v=bOigXb_9L28 (letzter Zugriff: 18.11.2020).

Forumbeitrag auf swtorcantina. http://swtorcantina. de/paketoeffnen-neu-gestaltet/ (letzter Zugriff: 18.11.2020).

Gogl, Ingrid/Lebersorger, Fabian (2016): Shitstormen auf Österreichisch \#oidaRP. Ber-lin. TIB AV-PORTAL: https://doi.org/10.5446/20833 (letzter Zugriff: 18.11.2020).

Kuhlhoff (2017): „Da kotze ich doch im Strahl” - Wie sieht die perfekte Wutrede aus? 11FREUNDE: https://11freunde.de/artikel/da-kotze-ich-doch-imstrahl/531888 (letzter Zugriff: 18.11.2020).

Leef, George (2014): "Hate Facts" - When the Truth is Intolerable. In: National Associa-tion of Scholars: https://www.nas.org/blogs/article/hate_facts_when_ the_truth_is_intolerable (letzter Zugriff: 18.11.2020).

Lindner, Christian: Die Wutrede von FDP-Chef Lindner. https://www.latest.facebook.com/WELTnext/ videos/788263411221273/ (letzter Zugriff: 18.11.2020).

o.V. (2014): Heißsporn Gattuso macht den Trappatoni. KURIER: https://kurier.at/sport/fussball/fussballheisssporn-gattuso-macht-den-trappatoni/87.672.696 (letzter Zugriff: 18.11.2020).o.V. (2019): Thunberg hält Wutrede bei UN - Merkel widerspricht ihr. Berliner Morgenpost: https://www.morgenpost. de/politik/article227181531/Greta-Thunberg-haeltWutrede-bei-UN-Trump-spottet-ueber-sie.html (letzter Zugriff: 18.11.2020).

Palmer, Boris: Facebook-Post. https://www.facebook.com/ ob.boris.palmer/posts/2381885385184313 (letzter Zugriff: 18.11.2020).

Sievert, Timo: Facebook-Seite. https://www.facebook. com/DerSievi/ (letzter Zugriff: 18.11.2020).

Sievert, Timo: Nach Nordderby-Niederlage: HSV-Fan platzt der Kragen - genial Wutrede. https://www. youtube.com/watch?v=e68-oPtCBfE (letzter Zugriff: 18.11.2020).

Syd (2014): Steinmeiers Wutrede als YouTube-Hit. SPIEGEL: https://www.spiegel.de/politik/ausland/ steinmeier-bruellt-montagsdemo-auf-alexanderplatzin-berlin-nieder-a-970571.html (letzter Zugriff: 18.11.2020).

SZ Magazin: Tweet. https://twitter.com/szmagazin/ status/977978031481540608 (letzter Zugriff: 18.11.2020).

Thomys, Alexander (2018): Özdemirs Wutrede sorgt für Begeisterung. SÜDWEST PRESSE: https://www. 
swp.de/suedwesten/landkreise/ermstal/oezdemirswutrede-sorgt-fuer-begeisterung-24857234.html (letzter Zugriff: 18.11.2020).

Tsp/rtr/epd/KNA (2014): Wutrede von Papst Franziskus: "Die Kurie ist krank". DER TAGESSPIEGEL: https:// www.tagesspiegel.de/gesellschaft/panorama/ wutrede-von-papst-franziskus-die-kurie-istkrank/11155134.html (letzter Zugriff: 18.11.2020).

Video Hallo, mein Name ist Paul Weston und ich bin ein Rassist. PI News: http://www.pi-news.net/2013/06/ video-hallo-mein-name-ist-paul-weston-und-ich-binein-rassist/ (letzter Zugriff: 18.11.2020).

Video Wutrede vom Wettpaten an Pep Guardiola !!!!. https://www.youtube.com/watch?v=OW-Ey36MsoM (letzter Zugriff: 18.11.2020).

Wegner, Nils (2017): Sezession 79 - das neue offene Heft! Sezession im Netz: https://sezession.de/57340/ sezession-79-das-neue-offene-heft (letzter Zugriff: 18.11.2020).

\section{Forschungsliteratur}

Aristoteles (2002): Rhetorik. Werke in deutscher Übersetzung Bd. 4. Übersetzt und erläutert von Christof Rapp. Berlin: Akademie-Verlag.

Auer, Katrin (2002): „Political Correctness" - ideologischer Code, Feindbild und Stigmawort der Rechten. In: Österreichische Zeitschrift für Politikwissenschaft 31/3, S. 291-303.

Ayaß, Ruth (2011): Kommunikative Gattungen, mediale Gattungen. In: Habscheid, Ste-phan (Hg.): Textsorten, Handlungsmuster, Oberflächen. Berlin/ Boston: De Gruyter, S. 275-295.

Boehnke, Lukas (2019): Rechter Kulturkampf heute. Identitätskonstruktion und Framing-Strategien der Identitären Bewegung. In: Boehnke, Lukas/Thran, Malte/Wunderwald, Jacob (Hgg.): Rechtspopulismus im Fokus. Theoretische und praktische Herausforderungen für die politische Bildung. Wiesbaden: Springer Fachmedien, S. 89-114.

Boyd, Danah (2014): It's complicated. The social lives of networked teens. New Haven: Yale University Press.

Briggs, Charles L./Bauman, Richard (1992): Genre, Intertextuality, and Social Power. In: Journal of Linguistic Anthropology 2/2, S. 131-172.

Brokoff, Jürgen/Walter-Jochum, Robert (2019): Verachtung und Hass aus literaturwissenschaftlicher Sicht. In: Kappelhoff, Hermann/Bakels Jan-Hendrik/ Lehmann Hauke et al. (Hgg.): Emotionen: Ein interdisziplinäres Handbuch. Stuttgart: J.B. Metzler, S. 225-229.

Bruns, Julian/Glösel, Kathrin/Strobl, Natascha (2017): Die Identitären. Rechtsextreme Ideologie der Neuen Rechten und modernisierter Rassismus einer Jugendbewegung. In: Jahrbuch für Pädagogik 2017/1, S. 81-102.

Burger, Harald/Luginbühl, Martin (2014): Mediensprache. Eine Einführung in Sprache und Kommunikationsformen der Massenmedien. 4. überarbeitete und erweiterte Auflage. Berlin/Boston: De Gruyter Mouton.

Deppermann, Arnulf (2008): Gespräche analysieren. Eine Einführung. Wiesbaden: VS.
DWDS ,Shitstorm': https://www.dwds.de/r?q=shitstormen\&corpus=ibk_web_2016c (letzter Zugriff: 18.11.2020).

Ellerbrock, Dagmar/Koch, Lars/Müller-Mall, Sabine et al. (2017): Invektivität - Perspektiven eines neuen Forschungsprogramms in den Kultur- und Sozialwissenschaften. In: Kulturwissenschaftliche Zeitschrift 2/1, S. 2-24.

Fiehler, Reinhard (1993): Grenzfälle des Argumentierens. "Emotionalität statt Argumentation" oder "emotionales Argumentieren". In: Püschel, Ulrich/ Sandig, Barbara (Hgg.): Stilistik. Bd. 3. Argumentationsstile. Hildesheim/New York: Olms, S. 149-174.

Goffman, Erving (1977): The Arrangement between the Sexes. In: Theory and Society 4/3, S. 301-331.

Günthner, Susanne (2002): Stimmenvielfalt im Diskurs. Formen der Stilisierung und Ästhetisierung in der Redewiedergabe. In: Gesprächsforschung 3, S. 59-80.

Günthner, Susanne/Knoblauch, Hubert (1994): ,'Forms are the food of faith". Gattungen als Muster kommunikativen Handelns. In: Kölner Zeitschrift für Soziologie und Sozialpsychologie 46/4, S. 693-723.

Habscheid, Stephan (2011): Das halbe Leben. Ordnungsprinzipien einer Linguistik der Kommunikation - Zur Einleitung in den Band. In: Habscheid, Stephan (Hg.): Textsorten, Handlungsmuster, Oberflächen. Linguistische Typologien der Kommunikation. Berlin/ Boston: De Gruyter, S. 3-30.

Hanks, William F. (1987): Discourse Genres in a Theory of Practice. In: American Ethnologist 14/4, S. 668-692.

Hiippala, Tuomo/Tseng, Chiao-I (2017): Editorial. Media evolution and genre expectations. In: Discourse, Context \& Media 20, S. 157-159.

Jauß, Hans Robert (1977): Theorie der Gattungen und Literatur des Mittelalters. In: Alterität und Modernität der mittelalterlichen Literatur. Gesammelte Aufsätze 1956-1976. München: Fink, S. 327-358.

Jones, Rodney (2018): Surveillant media. Technology, language, and control. In: Cotter, Colleen/Perrin, Daniel (Hgg.): The Routledge handbook of language and media. Milton Park et al.: Routledge.

Knoblauch, Hubert/Schnettler, Bernt (2010): Sozialwissenschaftliche Gattungsforschung. In: Zymner, Rüdiger (Hg.): Handbuch Gattungstheorie. Stuttgart/ Weimar: Metzler, S. 291-294.

Koch, Lars/Nanz, Tobias/Rogers, Christina (2020): The Great Disruptor. Eine Annäherung. In: Dies. (Hgg.): The Great Disruptor. Über Trump, die Medien und die Politik der Herabsetzung. Stuttgart: J.B. Metzler, S. 1-19.

Koster, Severin (1980): Die Invektive in der griechischen und römischen Literatur. Meisenheim am Glan: Hain.

Krieger, Manuela/Machnyk, Christina (2019): „Das Internet ist für uns alle Neuland." - Zur De- und Rekontextualisierung lexikalischer Einheiten in konventionalisierten Memes. In: Bülow, Lars/ Johann, Michael (Hgg.): Politische Internet-Memes - Theoretische Herausforderungen und empirische Befunde. Berlin: Frank \& Timme, S. 115-142.

Lehmann, Johannes F. (2019): Zorn und Wut im Spannungsfeld der Literaturgeschichte. In: Kappelhoff, Hermann/Bakels Jan-Hendrik/Lehmann 
Hauke et al. (Hgg.): Emotionen. Ein interdisziplinäres Handbuch. Stuttgart: J.B. Metzler, S. 180-184.

Leibniz-Institut für Deutsche Sprache (2020): Deutsches Referenzkorpus / Archiv der Korpora geschriebener Gegenwartssprache 2020-I (Release vom 21.01.2020). Mannheim: Institut für deutsche Sprache.

Lomborg, Stine (2011): Social media as communicative genres. In: MedieKultur. Journal of media and communication research 27/51, S. 55-71.

Marx, Konstanze (2017): Rekontextualisierung von Hate Speech als Aneignungs- und Positionierungsverfahren in Sozialen Medien. In: Aptum 13/2, S. 132-146.

Marx, Konstanze (2019): Von Schafen im Wolfspelz - Shitstorms als Symptome einer medialen Emotionskultur. In: Hauser, Stefan/Luginbühl, Martin/ Tienken, Susanne (Hgg.): Mediale Emotionskulturen. Bern: Peter Lang, S. 135-153.

Meier-Vieracker, Simon (2020): Selbstlegitimationen von Hass auf rechten Internetseiten. In: Kämper, Heidrun/Warnke, Ingo H. (Hgg.): Diskurs - ethisch. Bremen: Hempen, S. 139-155.

Meier, Simon (2016): Wutreden - Konstruktion einer Gattung in den digitalen Medien. In: Zeitschrift für germanistische Linguistik 44/1, S. 37-68.

Meier, Simon/Marx, Konstanze (2019): Doing genre in the digital media. In: Brock, Alexander/Pflaeging, Jana/Schildhauer, Peter (Hgg.): Genre emergence. Developments in print, TV and digital media. Frankfurt a.M./New York: Peter Lang, S. 191-212.

Meier, Simon/Viehhauser, Gabriel (2020): Rekontextualisierung als Forschungsparadigma des Digitalen? Einleitung in den Band. In: Dies./Sahle, Patrick (Hgg.): Rekontextualisierung als Forschungsparadigma des Digitalen. Norderstedt: Books on Demand, S. 1-20.

Miller, Carolyn R. (1984): Genre as social action. In: Quarterly Journal of Speech 70/2, S. 151-167.

Neumann, Uwe (1998): Invektive. In: Ueding, Gert (Hg.): Historisches Wörterbuch der Rhetorik. Bd. 4. Tübingen: Niemeyer, S. 549-561.

Niehr, Thomas/Reissen-Kosch, Jana (2018): Volkes Stimme? Zur Sprache des Rechtspopulismus. Berlin: Dudenverlag.

OWID ,Shitstorm': https://www.owid.de/artikel/402347 (letzter Zugriff: 18.11.2020).

Pflaeging, Jana (2020): Diachronic perspectives on viral online genres. From images to words, from lists to stories. In: Thurlow, Crispin/Dürscheid, Christa/Diémoz, Federica (Hgg.): Visualizing Digital Discourse. Berlin/Boston: De Gruyter, S. 227-244.

Pichl, Robert (1998): Iracundia. In: Ueding, Gert (Hg.): Historisches Wörterbuch der Rhetorik. Bd. 4. Tübingen: Niemeyer, S. 597-599.

Reisigl, Martin (2014): Gattung. In: Wrana, Daniel/Ziem, Alexander/Reisigl, Martin et al. (Hgg.): DiskursNetz. Wörterbuch der interdisziplinären Diskursforschung. Berlin: Suhrkamp, S. 158.

Sell, Aaron (2019): The Evolutionary Psychology of Anger. In: Kappelhoff, Hermann/Bakels Jan-Hendrik/ Lehmann Hauke et al. (Hgg.): Emotionen. Ein interdisziplinäres Handbuch. Stuttgart: J.B. Metzler, S. 174-179.

Spiegel, Carmen (2011): Streit. Eine linguistische Untersuchung verbaler Interaktionen in alltäglichen Zusammenhängen. Mannheim: Verlag für Gesprächsforschung.

Timmons, Travis (2018): Wutrede Hall of Fame. Giovanni Trapattoni, the Original. In: The Bundesliga Fanatic: https://web.archive.org/web/20180115001849/ http://bundesligafanatic.com/wutrede-hall-of-famegiovanni-trapattoni-the-original/ (letzter Zugriff: 28.11.2020).

Voßkamp, Wilhelm (1977): Gattungen als literarischsoziale Institutionen. In: Hinck, Walter (Hg.): Textsortenlehre - Gattungsgeschichte. Heidelberg: Quelle \& Meyer, S. 27-42.

Weiß, Christian (2005): Die thematische Erschließung von Sprachkorpora. Mannheim: Institut für deutsche Sprache.

West, Candace/Zimmerman, Don H. (1987): Doing Gender. In: Gender and Society 1/2, S. 125-151. 